\title{
ANALYSIS OF TWO GROUPS OF YOUNG PATIENTS WITH BREAST CANCER
}

\author{
Matheus de Paula Solino¹, Mariana Soares Cardoso¹, Marcelo Antonini \\ ${ }^{1}$ Instituto de Assistência Médica ao Servidor Público Estadual de São Paulo - São Paulo (SP), Brazil.
}

Introduction: Breast cancer is the main type of cancer and the main cause of death by cancer among women worldwide. For Brazil, the National Cancer Institute José Alencar Gomes da Silva (Instituto Nacional de Câncer José Alencar Gomes da Silva - INCA) estimated that breast cancer would be responsible for $29.5 \%$ of new cancer cases and $16.2 \%$ of cancer deaths in 2019. The incidence of breast cancer in young women has grown worldwide: in developing countries, $25 \%$ of breast cancer cases occur in women under 40 years of age. In young women, breast cancer has more aggressive characteristics and is diagnosed later. Mammography is the best screening method, however, it is only started at 40. Objective: To analyze the clinical, pathological, and treatment characteristics among patients with breast cancer diagnosed up to 40 years old and between 41 and 45 years old. Methods: Observational and cross-sectional study, which evaluated patients diagnosed with breast cancer until the age of 45 and who underwent surgical treatment at the Hospital do Servidor Público Estadual between October, 2013 and October, 2017. Data were collected from medical records and patients were divided into two groups: Group 01, up to 40 years old and Group 02, from 41 to 45 years old. Variables were collected regarding age at diagnosis, menarche, number of deliveries, body mass index (BMI), comorbidities, family history of breast cancer, initial clinical staging, type of biopsy, type of histological biopsy result, type of treatment, and surgical results. The variables were analyzed statistically. Results: Fifty patients aged between 29 and 45 years old were evaluated. There was no statistically significant difference in clinical characteristics. Group 02 presented more tumors with hormonal receptors, more cases of axillary emptying after compromised sentinel lymph node, and lower rates of radiotherapy. Conclusion: Young patients aged 41-45 years old present more tumors with hormonal receptors, greater involvement of microscopically locoregional lymph nodes, and less treatment with radiotherapy than young patients under 40 years of age. 